\title{
Dermatofibrosarcoma Protuberans - A Case Report
}

\author{
Raju Kamlakarrao Shinde, Sajika Pramod Dighe², Sangita Jogdand Shinde ${ }^{3}$, Anupam Anand ${ }^{4}$
}

1,2,4 Department of Surgery, Jawaharlal Nehru Medical College, DMIMS, Sawangi, Wardha, Maharashtra, India.

${ }^{3}$ Department of Pharmacology, Jawaharlal Nehru Medical College, DMIMS, Sawangi, Wardha, Maharashtra, India.

\section{PRESENTATION OF CASE}

A 74-year-old female presented with recurrent swelling over right sole of foot since 2 years; associated with pain and difficulty in walking. Within a span of 5 months the patient noticed progressive increase in the swelling hence, she underwent magnetic resonance imaging (MRI) foot which suggested nerve sheath tumour with possible malignant transformation and biopsy which was suggestive of spindle cell tumour mostly cellular neurofibroma.

On examination a swelling of $8 \times 5 \times 4 \mathrm{~cm}$ was present on the sole of the right foot more medial to the median arch, pinkish brown in colour, irregular borders, hard consistency and tender to touch with no evidence of popliteal and inguinal lymphadenopathy.

Dermatofibrosarcoma protuberans is an infrequent disease and reports for, $<0.1$ $\%$ of all malignancies and $<1 \%$ of all soft-tissue tumours with annual incidence of 4.2 per million. It is derived from dermal fibroblasts and is a superficial cutaneous low-grade malignancy, locally pugnacious with an increased rate of local recurrence but with a smaller risk of metastasis. It was $1^{\text {st }}$ reported by Hoffman in 1925 and there is no large-scale research performed to identify differences in incidence of dermatofibrosarcoma protuberans across race and sex. It clinically has a sluggish growth and has a low to moderate grade, occurring in middle-aged adults. Histology pictures show composition of unvaried spindle cell fascicles growing in storiform pattern with strong CD34 immunoreactivity. ${ }^{1,2}$

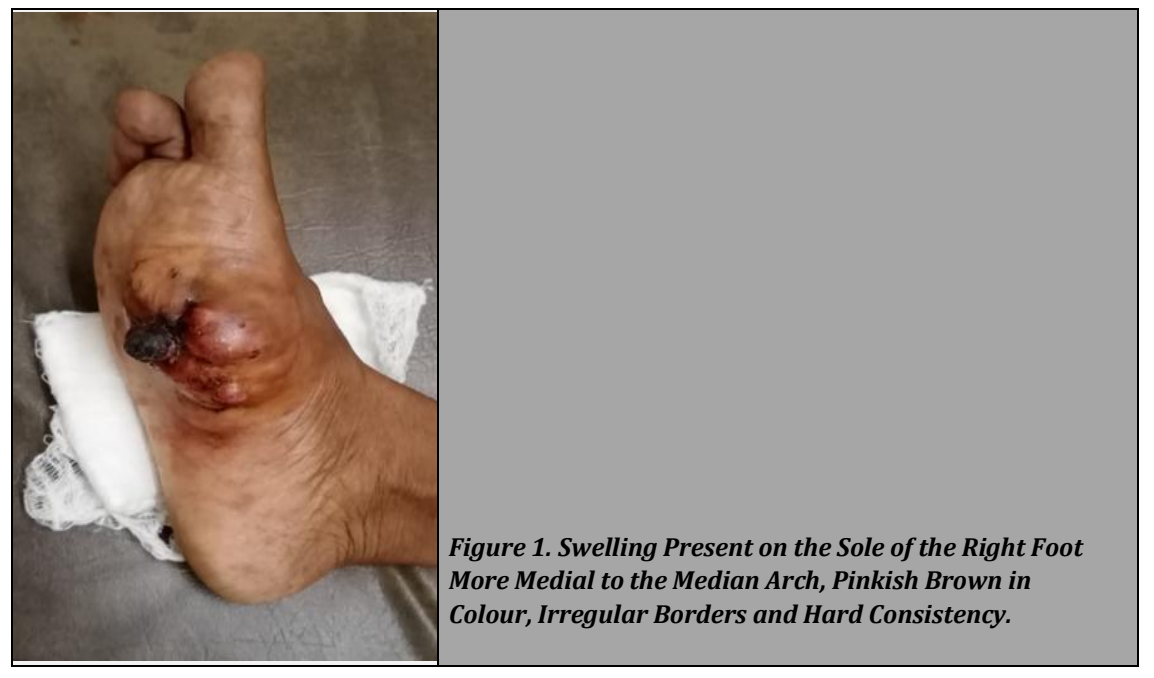

Computed Tomography (CT) foot was suggestive of schwannoma with erosion of underlying cortex of $1^{\text {st }}$ and $2^{\text {nd }}$ metatarsal and cuboid bones with lesion causing splaying and stretching of tibialis posterior, flexor hallucis longus, flexor digitorum longus tendons with involvement of tibial nerve and involvement of interposing fat planes with plantar muscles and extension up to cutaneous plane causing breech in plantar fascia and repeat biopsy suggestive of cellular neurofibroma.
Corresponding Author: Dr. Sajika Pramod Dighe, Department of Surgery, Jawaharlal Nehru Medical College, DMIMS, Sawangi, Wardha, Maharashtra, India.

E-mail: sajikadighe@gmail.com

DOI: $10.14260 /$ jemds/2021/13

How to Cite This Article: Shinde RK, Dighe SP, Shinde SJ, et al. Dermatofibrosarcoma protuberans - a case report. I Evolution Med Dent Sci 2021;10(01):65-67, 10.14260/jemds/2021/13

Submission 14-09-2020,

Peer Review 08-11-2020,

Acceptance 14-11-2020,

Published 04-01-2021.

Copyright (C) 2021 Raju Kamlakarrao Shinde et al. This is an open access article distributed under Creative Commons Attribution License [Attribution 4.0 International (CC BY 4.0)] 


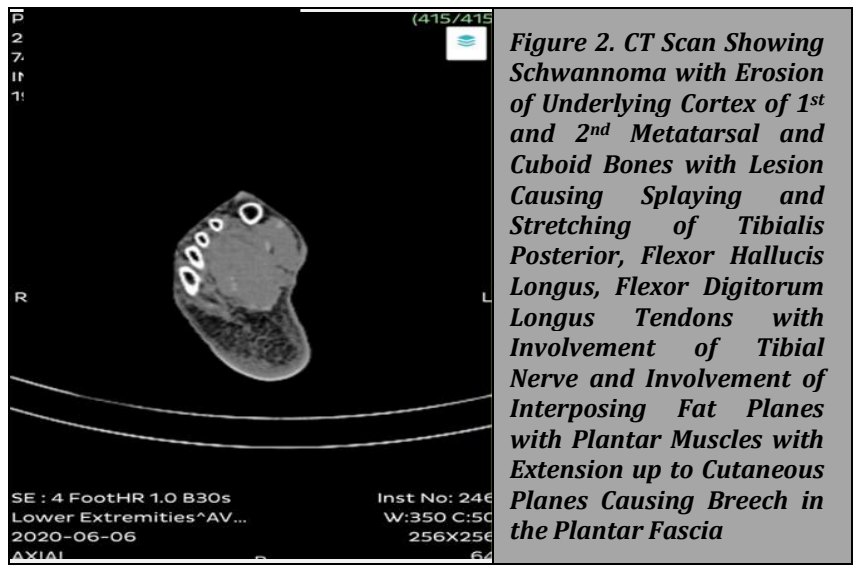

Chest x-ray was normal with no evidence of any other site of metastasis. Patient was advised transposition flap but was not willing for it. Hence, on the basis of patient's age, clinical condition and patient's unwillingness to follow-up, a decision of below knee amputation was taken.

Histopathology report post-operatively revealed dermatofibrosarcoma protuberans with immunohistochemical CD34 and S-100 positive, smooth muscle actin (SMA) and Vimentin negative.

She was advised to take prophylactic imatinib mesylate for 2 years with follow-up after 6 months.

\section{DISCUSSION}

Dermatofibrosarcoma protuberans is clinically scarce and unique hence, needs a high index of clinical suspicion as it is difficult to diagnose. The differentials include neurofibroma, leiomyoma, malignant melanoma, morpheaform basal cell carcinoma, keloid, desmoid tumours, Kaposi sarcoma, fibrosarcoma, dermatofibroma, nodular fasciitis, cellular blue nevus, pleomorphic sarcoma of the skin, leiomyosarcoma, malignant peripheral nerve sheath tumours, sarcoidosis and rare variants of spindle cell malignant melanoma. 1,2,3,4 $^{2}$

Clinically it has sluggish growth, low to moderate grade and commonly occurs between $2^{\text {nd }}$ to $5^{\text {th }}$ decade of life with equal predilection in males and females with occurrence in children as well. More commonly occurs of on trunk (40 - 50 $\%$ ), chest and shoulders (30 - $40 \%$ ) and proximal portion of limbs (10 - $15 \%)$ head and neck (10 - $15 \%)$ and rare sites includes toes, scalp, breast and vulva. Provisional diagnosis can be made on the basis of clinical appearance, location and characteristic findings. In premature stage of dermatofibrosarcoma protuberans, the patient detects a small, sluggishly growing, firm, painless, skin-coloured dermal plaque with or without subcutaneous thickening or nonprotuberant lesion. This early lesion gradually progresses into protuberant stages, and if left untreated can locally invade into fascia, muscle, periosteum, bone and in smaller number of cases can metastasize to other organs in advanced stages. Common sites of metastasis are lung, brain, bone, visceral organs, lymph-nodes and soft tissues. This activity is insidious and takes several months to years to complete. Because of its low incidence it is always missed or under-diagnosed and is conventionally slow growing flesh coloured or slightly yellowbrown tumour without epidermal invasion but subcutaneous spread. A sudden swift increase denotes fibro-sarcomatous metamorphosis. It can be associated with purulent exudate or ulceration and pain as then lesion progresses ${ }^{1,2,4,5}$

The cornerstone of definitive identification is based on thorough history, clinical examination, suspicion of dermatofibrosarcoma protuberans and chiefly 'gold standard' biopsy (incisional, punch or excisional) to confirm the pathological diagnosis. Lymph-node examination and imaging studies are important for drawing a surgical plan and staging. As of now a conventional staging system has not been described in literature but commonly primary tumour is described as stage I, lymph-node metastasis as stage II and distant metastasis as stage III. "A recent report by Hao X et al. gave a staging system on the basis of European consensus based interdisciplinary guidelines as follows": 2,4

\begin{tabular}{|c|c|}
\hline Stage & Criteria \\
\hline Stage I & $\begin{array}{l}\text { Non-protuberant lesion including atrophic or sclerotic plaque, } \\
\text { macula or small nodules }\end{array}$ \\
\hline Stage II & Protuberant primary tumour \\
\hline Stage IIA & Superficial tumour : without invasion of underlying fascia \\
\hline Stage IIB & $\begin{array}{l}\text { Deep tumour : either superficial to the fascia or occurred beneath the } \\
\text { superficial fascia }\end{array}$ \\
\hline Stage III & Lymph node metastasis \\
\hline Stage IV & Distant metastasis to other organs \\
\hline \multicolumn{2}{|c|}{ Table 1. Depicting Stages of Dermatofibrosarcoma Protuberans } \\
\hline
\end{tabular}

Small lesions with no other clinical findings of metastasis can be managed without imaging studies though radiological evaluation provides better demarcation of local extension of the disease thus providing accurate surgical planning. MRI and computed tomography both can be done as they invariably reveal soft tissue mass which enhances on contrast. MRI though has an upper hand in defining extent of tissue infiltration, margin and depth of involvement of underlying fascia and musculature which helps in surgical planning and selecting most appropriate reconstruction strategy as well can be used in post-operative recurrence surveillance as can contribute to distinguish between postoperative alterations, scars vs. recurrence. Also it help in defining the atypical cases and involvement of critical structure. MRI is now consistently used in following conditions namely: large tumour size, tumours with sceptical deeper component, recurrent tumours, critical anatomic locations and re-excision of dermatofibrosarcoma protuberans with positive margins. Other techniques used are fluorodeoxyglucose - positron emission tomography (FDG-PET) to identify metastatic disease and evaluation of treatment response. X-rays has no role in evaluation of primary tumour. In our case we have done MRI as well as CT for better demarcation of the tumour and its surrounding structure.1,2,6

Treatment options are surgical, radiation therapy and chemotherapy based on tumour anatomy and location. The primary apprehension in dermatofibrosarcoma protuberans is local recurrence due to its locally aggressive nature. The main aim of surgical intervention is resection to achieve negative margins. If initial surgery reports invasion or positive margins, re-resections is recommended until achievement of clear margins. Before definitive reconstruction, complete assessment of all margins is necessary. Surgical removal is a standard form of management in cases of dermatofibrosarcoma protuberans in all stages from I to IV. Types of surgical management includes wide local excision (WLE) with tumour or Mohs micrographic surgery (MMS) and partial or total amputation according to tumour site. The rate 
of localised recurrence with R0 resection is between 0.5 to 19 $\%$. At present, a standard recommendation is not available though a 2 to $4 \mathrm{~cm}$ margin is practiced as approved by National Comprehensive Cancer Network (NCCN) (2020) except when tissue is limited as in head and neck lesions. Multiple researches have reported that Mohs micrographic surgery has lower risk of recurrence as compared to wide local excision and was $2.72 \%$ and $9.10 \%$ respectively as according to data from Medline between 2008 to 2018. Hence, Mohs micrographic surgery is considered as treatment of choice as it is precise and gives accurate evaluation of surgical margins during excision with optimal cosmetic reconstruction and functional recovery but has a limited application since it needs special expertise for Mohs surgeons and needs co-ordination with histo-technologist as well as is done in step-wise pattern and is time consuming and labour intensive with higher cost for the patient. As compared to wide local excision it is comparatively easier and widely used techniques by majority of surgeons with immediate wound repair and is cost effective with good cosmetic results, but can have a higher rate of local recurrence. Limb-salvage is the main priority considered and amputation is the last resort done after assessment of all possibilities of potential treatment and reconstructive options. Amputation is considered when the tumour is encasing neurovascular structures and when reconstruction is not possible with severe functional compromise. In our case, we considered amputation because of involvement of surrounding muscles, nerves and erosion of bone with unwillingness of patient for follow-up thus making limb salvage impractical. External beam radiotherapy is mainly a treatment of choice for unresectable disease and adjuvant therapy after resection. External beam radiotherapy is advocated in some cases of recurrent dermatofibrosarcoma protuberans and in positive margins when $\mathrm{R} 0$ re-excision is unobtainable. Chemotherapy can be also administered in form of tyrosine kinase inhibitors i.e., imatinib is advised for management of inoperable primary tumour, locally inoperable recurring disease, immensely difficult-to-operate tumours for pre-operative reduction of tumour size and metastatic dermatofibrosarcoma protuberans with response in $50 \%$ of treated patients. ${ }^{2,4,6,7}$

\section{CONCLUSIONS}

Dermatofibrosarcoma protuberans though a rare potential malignancy, needs prompt evaluation and treatment due to its high morbidity and local recurrence. As in our case, in grave circumstances in view of patient compliance along with location of tumour, amputation is the most favoured surgical intervention.

Financial or other competing interests: None.

Disclosure forms provided by the authors are available with the full text of this article at jemds.com.

\section{REFERENCES}

[1] Wales C, Caravaglio JV, Radi M, et al. A rare case of dermatofibrosarcoma protuberans of the forefoot. Radiol Case Rep 2016;11(2):102-5.

[2] Hao X, Billings SD, Wu F, et al. Dermatofibrosarcoma protuberans: update on the diagnosis and treatment. J Clin Med 2020;9(6):1752.

[3] Dupree WB, Langloss JM, Weiss SW. Pigmented dermatofibrosarcoma protuberans (Bednar tumor). A pathologic, ultrastructural and immunohistochemical study. Am J Surg Pathol 1985;9(9):630-9.

[4] Saiag P, Grob JJ, Lebbe C, et al. Diagnosis and treatment of dermatofibrosarcoma protuberans. European consensusbased interdisciplinary guideline. European Journal of Cancer 2015;51(17):2604-8.

[5] LeBlanc J, Chan C, Zedlitz A. Dermatofibrosarcoma protuberans. Cutis 2017;100(1):E6-7.

[6] Reha J, Katz SC. Dermatofibrosarcoma protuberans. Surg Clin North Am 2016;96(5):1031-46.

[7] Ferrone ML, Raut CP. Modern surgical therapy: limb salvage and the role of amputation for extremity softtissue sarcomas. Surg Oncol Clin North Am 2012;21(2):201-13. 